\title{
Characterization of low frequency inter-ELM modes of H-mode discharges at ASDEX Upgrade
}

\author{
B. Vanovac ${ }^{1}$, E. Wolfrum ${ }^{2}$, M. Hoelzl ${ }^{2}$, M. Willensdorfer ${ }^{2}$, M. \\ Cavedon $^{2}$, G.F. Harrer ${ }^{3}$, F. Mink ${ }^{2,4}$, S.S. Denk ${ }^{2}$, S. Freethy ${ }^{2}$, M. \\ Dunne $^{2}$, P. Manz ${ }^{2}$, N C Luhmann $\mathrm{Jr}^{5}$ and the ASDEX \\ Upgrade Team \\ ${ }^{1}$ DIFFER - Dutch Institute for Fundamental Energy Research, De Zaale 20, 5612 AJ \\ Eindhoven, the Netherlands \\ ${ }^{2}$ Max-Planck-Institut fur Plasmaphysik, 85748 Garching, Germany \\ ${ }^{3}$ Institute of Applied Physics, TU Wien, Fusion@ÖAW, 1040 Vienna, Austria \\ ${ }^{4}$ Physik-Department E28, Technische Universität München, 85748 Garching, \\ Germany \\ ${ }^{5}$ Department of Electrical and Computer Engineering, University of California at \\ Davis, Davis, CA 95616, USA \\ E-mail: B.Vanovac@differ.nl
}

\begin{abstract}
The steep edge gradient region of tokamak plasmas in the high confinement regime is known to drive instabilities, which cause transport. Several diagnostics are used to allow for a high degree of characterization of low-frequency modes appearing in between type-I Edge Localizes Modes (ELMs). These modes are dominantly observed in Electron Cyclotron Emission (ECE) and ECE Imaging measurements as the modulation of radiation temperature $\left(\delta T_{\text {rad }}\right)$. In the radial magnetic field $\left(\dot{B}_{\mathrm{r}}\right)$ measurements, the frequency range of $4 \mathrm{kHz}$ to $12 \mathrm{kHz}$ is observed. The position of the mode is determined to be at the upper part of the steep gradient region, the poloidal mode velocity is changing from $1.5 \pm 0.5 \mathrm{~km} / \mathrm{s}$ to $2.5 \pm 0.5 \mathrm{~km} / \mathrm{s}$ and the toroidal mode number is 13 to 14 . A comparison with the measured $E \times B$ velocity leads to the conclusion that the phase velocity of the mode is smaller than $3 \mathrm{~km} / \mathrm{s}$ or zero. The poloidal structure of the modes is found to be in agreement with the poloidal structure size associated with $\mathrm{n}=13$ as estimated from the equilibrium calculations. The modes are compared between two different heating phases during one discharge, and are found to differ in duration, velocity, frequency and toroidal mode number. The possibility of non-linear interaction between these modes and other, high frequency modes existing in the narrow pedestal, is assessed via bicoherence. The presented analysis gives an unprecedented picture of the mode, its position, its structure and its velocity, calling for comparison with non-linear modelling.
\end{abstract}

\section{Introduction}

The improved confinement mode of tokamak operation, referred to as H-mode [1], is characterized by the formation of the Edge Transport Barrier (ETB) in the outermost 
part of the confined region. Characteristics for this region are steep gradients and the formation of a pedestal. The pedestal is, however, intermittently unstable and the ETB collapses in a quasi-periodic manner expelling heat and particles. These periodic events are called Edge Localized Modes (ELMs) [2, 3, 4]. The linear stability boundary, i.e., the onset of the ELM crash, is often described by the coupling of peeling and ballooning modes in the pedestal. The peeling modes are driven by local edge current density and the ballooning modes by the steep pressure gradient. When the pedestal parameters reach the peeling-ballooning limit, an ELM crash occurs [5]. However, before an ELM crash, there exists a broad spectrum of instabilities in the pedestal region, so-called interELM modes [6, 7]. These inter-ELM modes might cause transport and influence the pedestal structure. The pedestal structure determines the stability of coupled peelingballooning modes and therefore the achievable pedestal top pressure [8, 9]. As the core plasma is determined mainly by the pedestal height, fusion gain is directly related to the pedestal pressure [10]. The inter-ELM modes and precursors are focus of an ELM-directed research for decades. Both have been observed in different machines. MHD modes such as palm tree mode [11] and washboard modes [12] have been detected at JET. ELM precursors have been observed at ASDEX Upgrade [13], COMPASS-D [14] and JET [15]. At ASDEX Upgrade, simultaneous observations of high-frequency and low-frequency inter-ELM modes, related to type I ELMs, have been reported [16]. High $(200-300 \mathrm{kHz})$ and mid-frequency $(50-100 \mathrm{kHz})$ inter-ELM modes at ASDEX Upgrade have been characterized, and have been inferred from measurements of the toroidal and poloidal mode numbers to be resonant at q-surfaces near the minimum radial electric field $\left(E_{\mathrm{r}}\right)$ field and at the separatrix, respectively [7, 17]. Both frequency branches were measured with magnetic pick-up coils. The Electron Cyclotron Emission Imaging (ECEI) diagnostic also measures the inter-ELM modes in the pedestal, in the low-frequency part of the spectrum at ASDEX Upgrade [18, 19], and KSTAR [20]. With a careful analysis at the plasma edge, it is possible to distinguish between the different modes appearing in the narrow pedestal. To reliably employ Electron Cyclotron Emission (ECE) and ECE Imaging diagnostics, we perform forward modeling to account for the effect of the density variation onto radiation temperature at the plasma edge. This effect has been studied for ASDEX Upgrade [21, 22], for DIII-D [23], and for KSTAR [24]. This manuscript focuses on characterization of the low frequency ( $10 \mathrm{kHz}$ ) inter-ELM mode using edge measurements from several diagnostics at ASDEX Upgrade. The combination of spatial information, 2D structure, $q$ profiles, the profile of the $E_{\mathrm{r}}$, poloidal velocity, and toroidal mode numbers allow characterizing inter-ELM modes in great detail. Section 2 describes the set-up of the experiment necessary to study inter-ELM modes. Magnetic properties are discussed in section 3. Methods used for the localization of the mode are described in section 4. Poloidal structure and poloidal velocity from the ECEI system are presented in section 5. Possibilities for mode interaction are discussed based on bicoherence analysis of the data from the magnetics in section 6. A summary and discussion are given in section 7 . 


\section{Overview of the discharge}

The ASDEX Upgrade discharge \#34244, used for the analysis in this manuscript, is taken from a set of discharges designed to study the fluctuations in between ELMs. The parameters of this discharge are shown in figure 1. A moderate pedestal top density $n_{\mathrm{e}}^{\text {ped }}<5 \times 10^{19} \mathrm{~m}^{-3}$ is chosen to avoid cut-off of the ECE and ECEI. It is heated with low auxiliary power to obtain a low repetition frequency of ELMs $\left(f_{\text {ELM }} \sim 50 \mathrm{~Hz}\right)$, hence long inter-ELM periods. The constant toroidal magnetic field $B_{\mathrm{t}}=-2.5 \mathrm{~T}$ and the plasma current $I_{\mathrm{p}}=0.8 \mathrm{MA}$ are not shown. The $q_{95}$ is constant throughout the discharge. The outer plasma boundary $R_{\text {aus }}$ is varied to have a better resolution of edge diagnostics. The Neutral Beam Injection (NBI) power is increased at $6.1 \mathrm{~s}$ from $2.5 \mathrm{MW}$ to 3.4 MW. The Electron Cyclotron Resonance Heating (ECRH) of $1.2 \mathrm{MW}$ is constant during the phases of interest. For the analysis of the low-frequency inter-ELM modes and the evaluation of the radial electric field, we use two time windows. The radiated power is shown in the bottom plot and does not change significantly in between the two heating phases. We mark the first window as (I), with the auxiliary heating power of $3.7 \mathrm{MW}\left(\mathrm{P}_{\mathrm{NBI}}=2.5 \mathrm{MW}\right.$ and $\left.\mathrm{P}_{\mathrm{ECRH}}=1.2 \mathrm{MW}\right)$. The second time window (II) has auxiliary heating of $4.6 \mathrm{MW}\left(P_{\mathrm{NBI}}=3.4 \mathrm{MW}\right.$ and the $\left.P_{\mathrm{ECRH}}=1.2 \mathrm{MW}\right)$. The repetition frequency of ELMs $\left(f_{\mathrm{ELM}}\right)$ changes when going from phase one to phase two due to the difference in the input power. During phase one the $f_{\mathrm{ELM}}$ is $\sim 40 \mathrm{~Hz}$, and during the second phase, two frequency branches are detected, both with $f_{\mathrm{ELM}}$ below $100 \mathrm{~Hz}$. The time windows where we show individual modes measured in the magnetics are chosen around phases in which the outer plasma boundary $R_{\text {aus }}$ reaches its maximum value during one of its scans. Those time periods were selected because the magnetic measurements show a higher amplitude and hence a better signal due to the plasma being closer to the pick-up coils. There is no change in the signal quality of the other diagnostics when compared to the remainder of the time span in a constant heating phase. An example of different quantities characterizing the plasma edge during ELM cycles for the discharge \#34244 is given in figure 2. This time interval is chosen from the collection of time intervals where the Lithium Beam Emission Spectroscopy (Li-BES) operated in beam 'on' phase. The depicted time interval shows three ELM crashes. As an indication of an ELM crash, a peak in the divertor current that consists of the combined contributions of thermo-electric and Pfirsch-Schlüter currents [25], is used and is shown in figure 2(a). However, not only the divertor shunt current but all the other quantities exhibit an abrupt behaviour during an ELM crash. Figure 2(b) displays a Li-BES measurement [26] of the electron density at the pedestal top. A similar feature observed in the electron temperature we show in figure 2(c). The $T_{\mathrm{e}}$ measurements are performed with the Electron Cyclotron Emission (ECE) diagnostic [27]. Power spectral densities of the magnetic and ECE signals are presented in figures 2(d), 2(e) and 2(f), respectively. The recovery of plasma edge parameters follows the ELM crash. Soon after the crash, the pedestal density and temperature profiles start to build up, albeit on different time scales [28]. The electron density recovers at a faster rate whilst the 

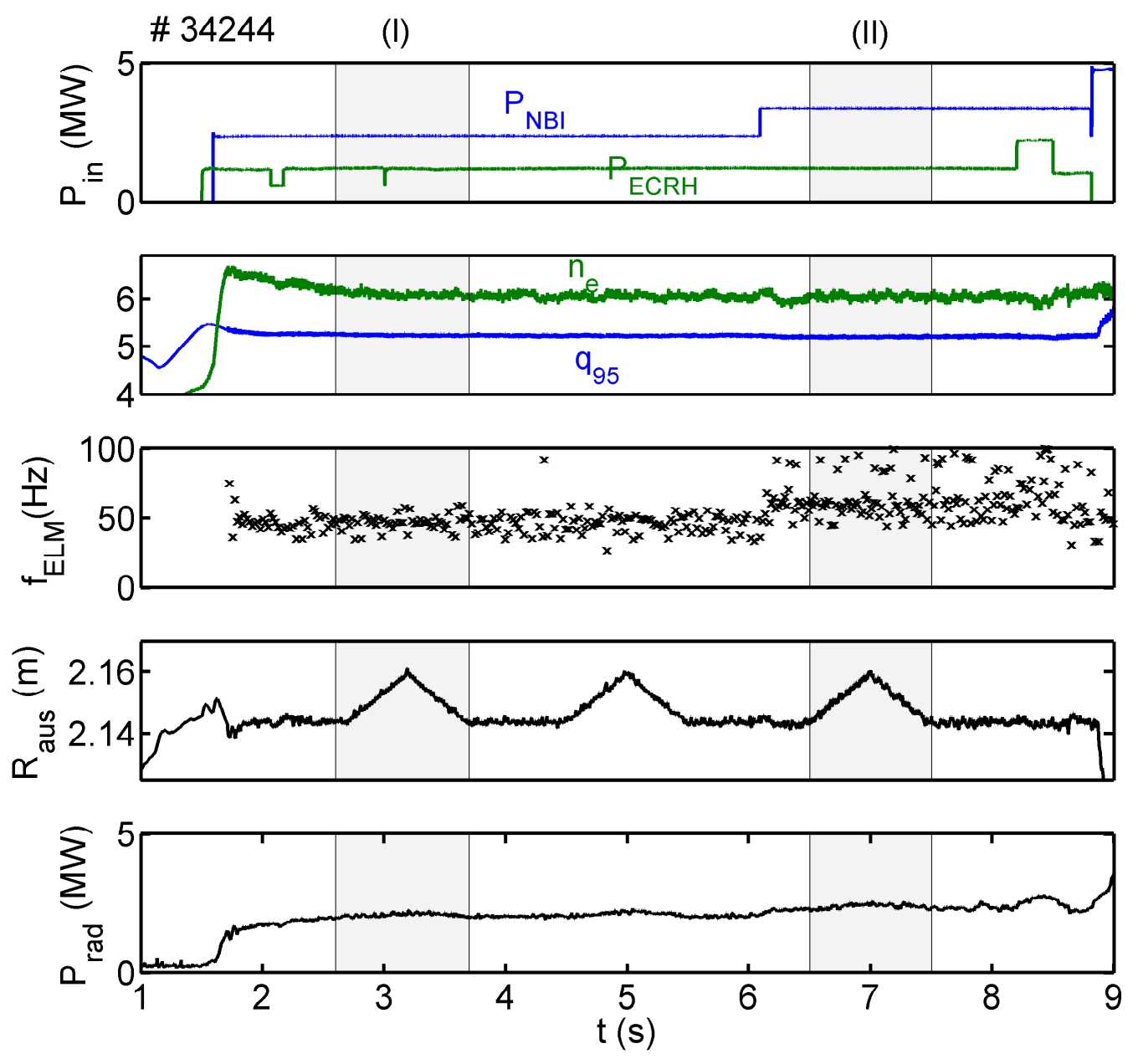

Figure 1. Overview of the parameters for discharge \#34244. From top to bottom: Auxiliary heating power, density and $q_{95}$, ELM frequency, position of the outer plasma boundary $\left(R_{\text {aus }}\right)$ and radiative power. The phases used for the analysis of inter-ELM periods, and for comparison of the radial electric field are marked as (I) and (II), respectively.

temperature increases gradually until the next ELM crash. From the spectrograms of magnetic and ECE data, we identify different magnetohydrodynamic (MHD) activities throughout an ELM cycle. MHD modes accompany an ELM crash [29]. While the edge electron density $\left(n_{\mathrm{e}}\right)$ and electron temperature $\left(T_{\mathrm{e}}\right)$ profiles recover, the electromagnetic signal is either quiet or shows the electromagnetic activity of very low amplitude. As shown in figure $2(\mathrm{~d})$, after the $T_{\mathrm{e}}$ recovery, the power spectral density of the magnetic signal peaks at a few frequency branches. The high frequency branch, studied in [6, 7] is of the order of $200 \mathrm{kHz}$, with identified toroidal mode numbers of $\mathrm{n} \sim 11$ [7]. This frequency branch is localized close to the minimum in $E_{\mathrm{r}}$, and the modes rotate with the velocity of the background $E \times B$ flow at that position in the electron diamagnetic direction. The mid-frequency branch ( $\sim 50 \mathrm{kHz}$ to $100 \mathrm{kHz})$ is short-lived. It appears once the pressure gradients are established and clamped. They are characterized by low 


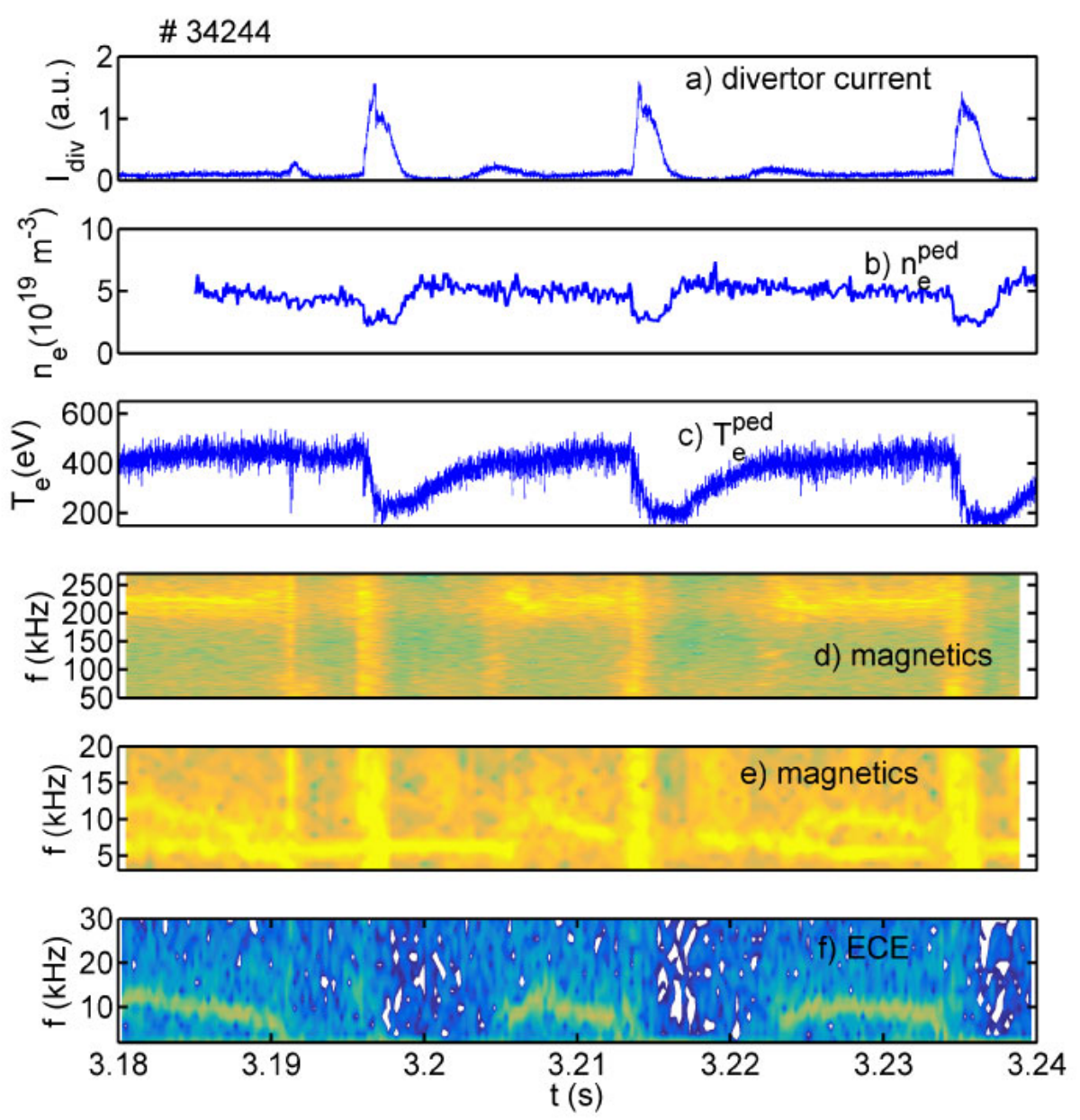

Figure 2. ELM cycles at ASDEX Upgrade: An example of the temporal evolution of different edge quantities during the discharge \#34244. a) Divertor shunt current as an ELM signature. b) Pedestal electron density measured with the Lithium Beam Emission Diagnostic. c) Pedestal electron temperature measured with the ECE radiometer. Spectrogram of the signal measured with a magnetic coil is displayed separately for the mid- to high- frequency range $(50 \mathrm{kHz}$ to $250 \mathrm{kHz})$ as shown in d) and in the low frequency range ( $4 \mathrm{kHz}$ to $20 \mathrm{kHz})$ as shown in e). f) Spectrogram of an ECE edge channel.

toroidal mode numbers of $n=4-6$ [17]. In the lowest part of the frequency spectrum a low frequency mode $\sim 10 \mathrm{kHz}$, as shown in the ECE power spectrum, is observed. This is the only MHD activity clearly visible on both ECE (figure 2(e)) and ECEI diagnostics during the inter-ELM period. The sampling rate of the ECE is $1 \mathrm{MHz}$ and the one of the ECEI is $500 \mathrm{kHz}$. The observation on the low-frequency modes have previously been reported in [22] and are measured as modulation in electron density, temperature and as MHD oscillations in Mirnov coils that measure the radial component of the magnetic field. In this manuscript only data from the magnetics, ECE and ECEI are 
analysed. The modes are, however, not always visible in the magnetic measurements and are obscured by a strongly visible core mode $(\sim 5 \mathrm{kHz}$ in this experiment) or its first harmonic that is of a similar frequency as that of the low-frequency mode. Bandpass or low pass filtering to the ECE and ECEI data is applied in the analysis, and is specified when performed in the further sections. As the visibility of the low frequency mode in the magnetic spectrogram increases with the plasma being closer to the wall we make use of the scan of the outer plasma boundary to analyze the data from the magnetics, as already suggested earlier in this manuscript.

\section{Magnetic properties of modes}

Figure 3 shows measurements of the magnetic pick-up coil and the ECE diagnostic during the inter-ELM period for two isolated inter-ELMs in different heating phases, labelled as (I) and (II) as indicated in figure 1. The measurements are performed at the outboard midplane. No filtering to the data was applied. The top frame of figure 3(a) displays the high-frequency part in the magnetic measurements, while the middle one depicts the low-frequency region of the same magnetic signal. The bottom frame shows the low-frequency part of the spectrum measured by a single edge channel of the ECE diagnostic. The high-frequency branch starts as a broad mode in the frequency range of $200-220 \mathrm{kHz}$ with toroidal mode number $\mathrm{n}=-8$ and continues its evolution as a mode with more localized frequency at around $220 \mathrm{kHz}$ with toroidal mode number of $\mathrm{n}=-9$. The minus sign describes the rotation of the modes in the electron diamagnetic direction (or counter-current direction). The low-frequency branch in the magnetic measurements displays the core mode with toroidal mode number $n=1$ and the edge mode with strong indication of $n=-13$. The frequency of the edge mode changes. It starts at $8 \mathrm{kHz}$, subsequently increasing to $11 \mathrm{kHz}$, and then slightly decreases until it is interrupted by an ELM crash. A similar behaviour is observed during the second heating phase as shown in figure $3(\mathrm{~b})$. The top frame shows the high-frequency branch of the magnetic signal with a single mode of frequency around $220 \mathrm{kHz}$ and the toroidal mode number $\mathrm{n}$ $=-9$. The middle frame shows the core $\mathrm{n}=1$ mode with a frequency of $9 \mathrm{kHz}$ and the edge mode with starting frequency of $7 \mathrm{kHz}$ that is decreasing to $4 \mathrm{kHz}$ just before an ELM crash. The toroidal mode number associated with the low-frequency edge mode is $\mathrm{n}=-14$. The low-frequency modes differ for the two heating phases. We observe changes in the frequency of the mode, toroidal mode number, and its lifetime. The frequency of the mode decreases by a factor of 2 as the input power increases. Interestingly, for both phases a 'slowing-down' pattern is observed. The change in toroidal mode number $\mathrm{n}$ from -13 to -14 could point towards a change in the location or change in the poloidal structure of the mode. The lifetime of the low-frequency mode shortens with an increase in the input power. In the low heating phase, the duration of the mode is about $10 \mathrm{~ms}$, while in the latter stage it decreases to about $6 \mathrm{~ms}$. However, in the second phase the ELM frequency increases, therefore, the ELM cycle shortens on account of the phase of clamped gradients. Contrary to the low-frequency modes, the high-frequency modes 

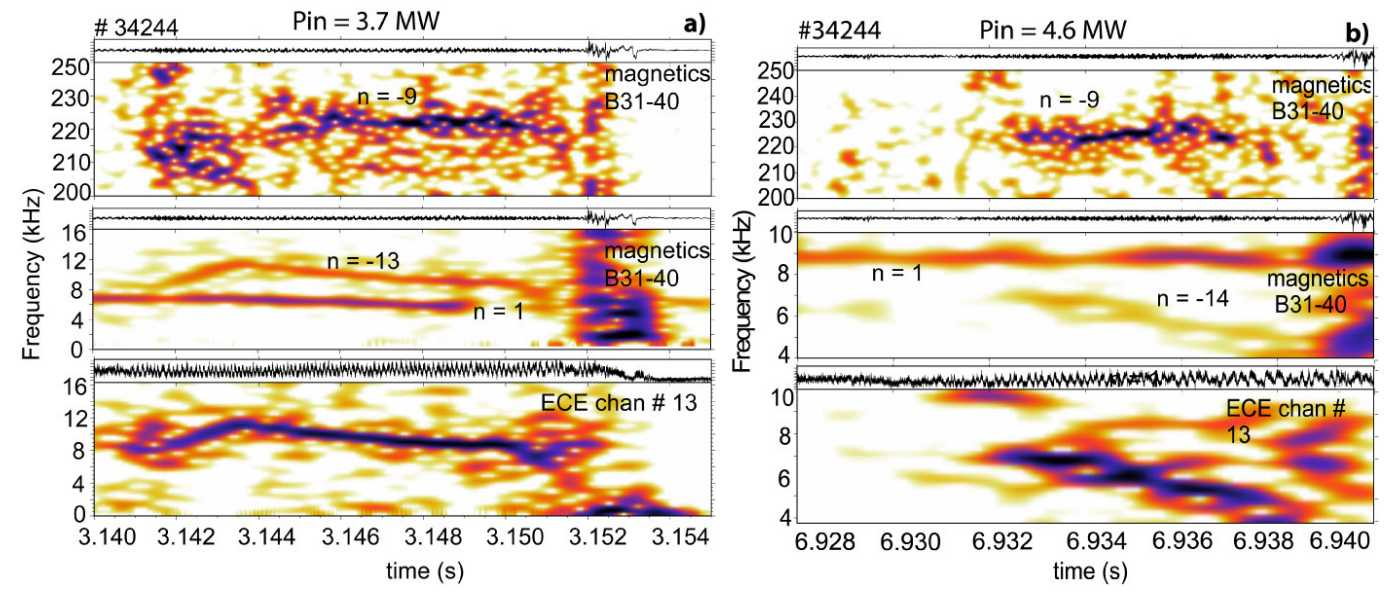

Figure 3. Spectrograms of magnetic (B31-40) and ECE (channel \#13) measurements during an inter-ELM period for lower (a) and higher (b) heating phase of the discharge \#34244 with respective toroidal mode numbers. A high-frequency mode appears as the fluctuation in the radial magnetic field. The low-frequency modes are measured as amplitude modulation in density, temperature and radial magnetic field.

do not change their frequency. They remain locked at $220 \mathrm{kHz}$ in both heating phases. The lifetime of the high-frequency modes is shorter in the second phase. It is of interest to note that the core mode changes in frequency from 7 to $9 \mathrm{kHz}$ with an increase in the input power as the plasma rotation increases.

\section{Localization}

For the localization of the low-frequency modes in the pedestal, we use measurements of the ECE diagnostics with a spatial resolution of about $6 \mathrm{~mm}$. With the Electron Cyclotron Radiation transport forward model [30,31] we determine the radial extent of the plasma contributing to the measured signal for each radial channel determined by the birth-place distribution function $\left(D_{\omega}\right) . D_{\omega}$ is defined as the normalized product of the emissivity $j_{\omega}$ and transmittance $T_{\omega}$, normalized to its integral representation of the radiation transport equation between the starting $s_{1}$ and end point $s_{2}$ along the line of sight [30]:

$$
D_{\omega}=\frac{j_{\omega} T_{\omega}}{\int_{s_{1}}^{s_{2}} j_{\omega} T_{\omega}}
$$

The radial resolution of a single channel is determined as the FWHM of $D_{\omega}$ and its measurement position, so-called 'warm resonance', is determined at the peak of $D_{\omega}$. The plasma edge, is a narrow region of about $2 \mathrm{~cm}$ inside the separatrix. Therefore, to accurately diagnose it, a high spatial resolution of any suitable diagnostic is required in this configuration. With the ECE channels, whose spatial resolution is about $6 \mathrm{~mm}$ at best, the gradient region is covered by at least three channels, depending on the configuration. The ECE Imaging on the other hand, with its radial resolution of about 
$1.5 \mathrm{~cm}$, comprises almost an entire gradient region within a single channel which is insufficient for precise radial localization. Its main feature, however, is the capability of poloidally resolved measurements (see section 5). Hence, for the localization we use measurements of the ECE diagnostic. On the top panels of figure 4 we show the radial contribution of the plasma to the measured signal for four adjacent ECE channels. The positions correspond to the peaks of $D_{\omega}$ evaluated for each channel. The four channels are shown together with the $T_{\mathrm{e}}$ profiles 4(a) and $n_{\mathrm{e}}$ profiles 4(b) obtained with Integrated Data Analysis (IDA) within the framework of Bayesian probability theory [32]. We show the mean profiles during an inter-ELM interval between $3.145 \mathrm{~s}$ to $3.15 \mathrm{~s}$. The selected channels are distributed with respect to the density profile as shown in
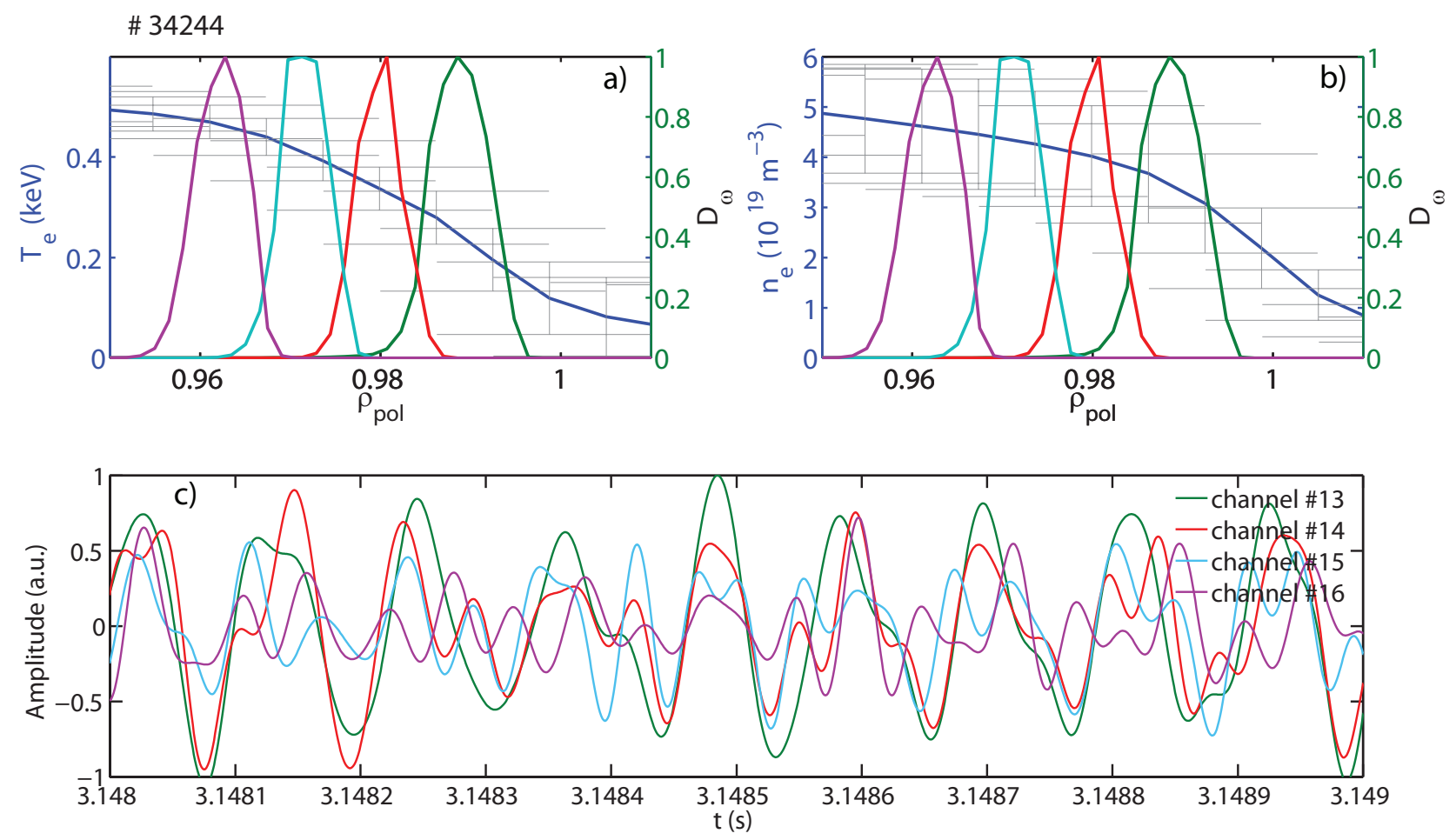

Figure 4. Phase relation between the four neighbouring ECE channels measuring in the pedestal. The birthplace distribution of the observed intensity $\left(D_{\omega}\right)$ together with $T_{\mathrm{e}}(\mathrm{a})$ and $n_{\mathrm{e}}(\mathrm{b})$ profiles. c) Time traces of filtered data measured with those four channels.

figure 4(b). The outermost channel \#13 measures at $\rho_{\text {pol }} \sim 0.99$. Channel \#14 is at the position $\rho_{\text {pol }} \sim 0.98$ and channel \#15 is further inside the pedestal top at the $\rho_{\text {pol }} \sim 0.97$ location. In channel \#16 at $\rho_{\mathrm{pol}} \sim 0.96$ the coherent modulation is vanishing. When compared to the temperature profiles (see figure 4(a)), this translates into a region from the steepest gradients until the temperature pedestal top. The signals measured by those four channels are overlaid in figure 4(c) to reveal their relative phase during the $1 \mathrm{~ms}$ time interval. Here, a band-pass Fourier filter $(7 \mathrm{kHz}$ to $25 \mathrm{kHz})$ is applied to the raw data. The correlation between the channels is given in the form of a correlation 
matrix shown in table 1.

\begin{tabular}{|c|c|c|c|c|}
\hline$\rho_{\text {pol }}($ chan \#) & $0.96(16)$ & $0.97(15)$ & $0.98(14)$ & $0.99(13)$ \\
\hline $0.96(16)$ & 1 & 0.25 & 0.267 & 0.264 \\
$0.97(15)$ & 0.25 & 1 & 0.490 & 0.397 \\
$0.98(14)$ & 0.267 & 0.490 & 1 & 0.81 \\
$0.99(13)$ & 0.264 & 0.397 & 0.81 & 1 \\
\hline
\end{tabular}

Table 1. The correlation matrix between four adjacent channels.

Figure 5(a) is showing a relative amplitude modulation $(\delta T / T)$ measured with ECE and averaged over the phase of constant frequency during the low-frequency mode. Depicted is the $8.8 \mathrm{kHz}$ component of the signal, alongside with the neighbouring $6 \mathrm{kHz}$ frequency displayed as a green shaded area representing the noise level. As shown, the fluctuation levels for channels \#13, \#14, \#15 is above the noise level with the highest fluctuation amplitude of $14 \%$ measured in the channel \#13. Channel \#16 is within the noise of the system. The magnitude of the measured fluctuations cannot be attributed to the electron temperature fluctuations alone, as a large contribution to $\delta T / T$ originates from the density modulation. This is shown in [21] where $5 \%$ of $\delta T / T$ in the region of steep gradients is a consequence of a change in the position of the channel due to the density modulation of $10 \%$. Hence, for an accurate amplitude estimate, we would need absolute values of the density fluctuations associated with this mode in combination with the radiation transport forward model. The relative phase between the channels is depicted in $5(\mathrm{~b})$. We do not observe a phase difference between the channels. However, one should be careful when evaluating the relative phase in the presence of density perturbation as the outward shift due to the density can influence the analysis. From the ECE measurements alone, it is only possible to say that the location of the mode is the upper corner of the steep gradient region, towards the pedestal top of the density. The amplitude itself is not sufficient for the mode width as small displacements in the steep gradient region will lead to significant relative amplitudes.

\section{Mode structure and poloidal velocity of the mode}

The poloidal velocity and the local poloidal wavelength can be determined from the measurements of poloidally distributed channels of the ECE Imaging system. For determination of a spatial structure and poloidal velocity, we use vertically distributed channels aligned along a single flux surface. The corresponding positions are obtained from the electron cyclotron radiation transport forward model. We compare the two different heating phases. This time, we chose two isolated inter-ELM periods, each corresponding to a certain heating phase during which the plasma boundary is kept at the same position. This ensures the stationary position of the mode during measurements. The temporal evolution of the poloidally distributed channels, above 

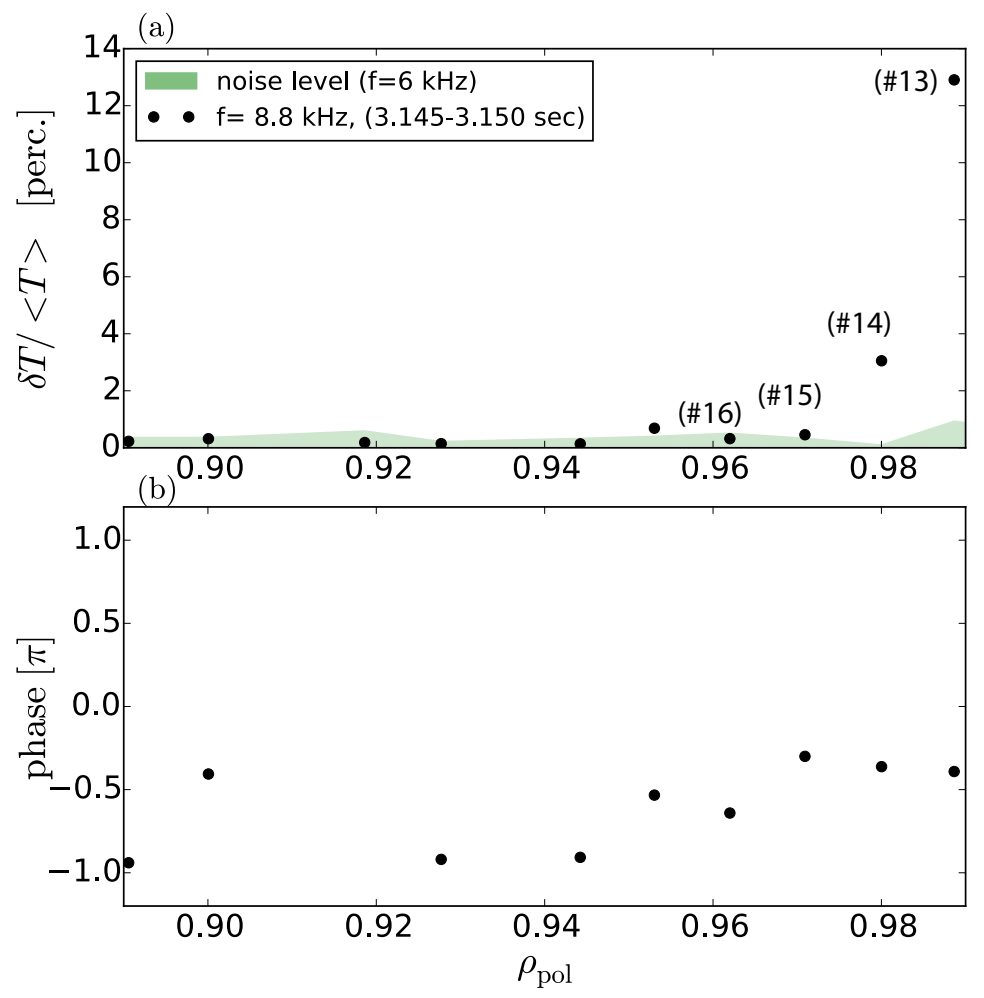

Figure 5. Shot \#34244. a) Amplitude of the edge ECE channels averaged over one inter-ELM cycle. b) The phase relation between neghbouring chanels.

the midplane, for shot \#34244 is displayed in figure 6. The upper image shows the mode propagation for the low heating and the bottom one, for the higher heating phase. In this representation, the mode is depicted as a structure (stripe) propagating from the bottom to the top in both cases. In the lab frame, this corresponds to the propagation in the electron diamagnetic direction and is in-line with the negative sign of the mode numbers calculated from the magnetic measurements shown in section 3, schematically indicated with the black straight lines shown in figure 6. The apparent mode velocity measured by ECEI for the phase I is $v_{\text {mode }} \sim 2.5 \pm 0.5 \mathrm{~km} / \mathrm{s}$, and for the phase II is $v_{\text {mode }} \sim 1.5 \pm 0.5 \mathrm{~km} / \mathrm{s}$. These velocities can be compared with the $E \times B$ flow at the position of the modes. As the mode rotates with the velocity that consists of the velocity of the background flow and the intrinsic phase velocity, $v_{\text {mode }}=v_{\mathrm{E} \times \mathrm{B}}+v_{\mathrm{ph}}$, the comparison would address the phase velocity of the mode. The $\mathrm{E} \times \mathrm{B}$ velocity is obtained as $v_{\mathrm{E} \times \mathrm{B}}=E_{r} / B$ where the $E_{\mathrm{r}}$ is measured with edge Charge Exchange Recombination Spectroscopy [33]. For the case of discharge \#34244, the measured $E \times B$ velocity profiles are shown in figure 7 for the two different heating phases. Here we show ELM synchronized data for the two phases labelled in figure 1. Note that the $E_{\mathrm{r}}$ measurements are ELM-synchronized in the time intervals of the scan of the outer plasma boundary. This significantly improves the radial resolution of the system. The radial span of the plasma, corresponding to the ECEI measurements from $\rho_{\text {pol }}$ between 

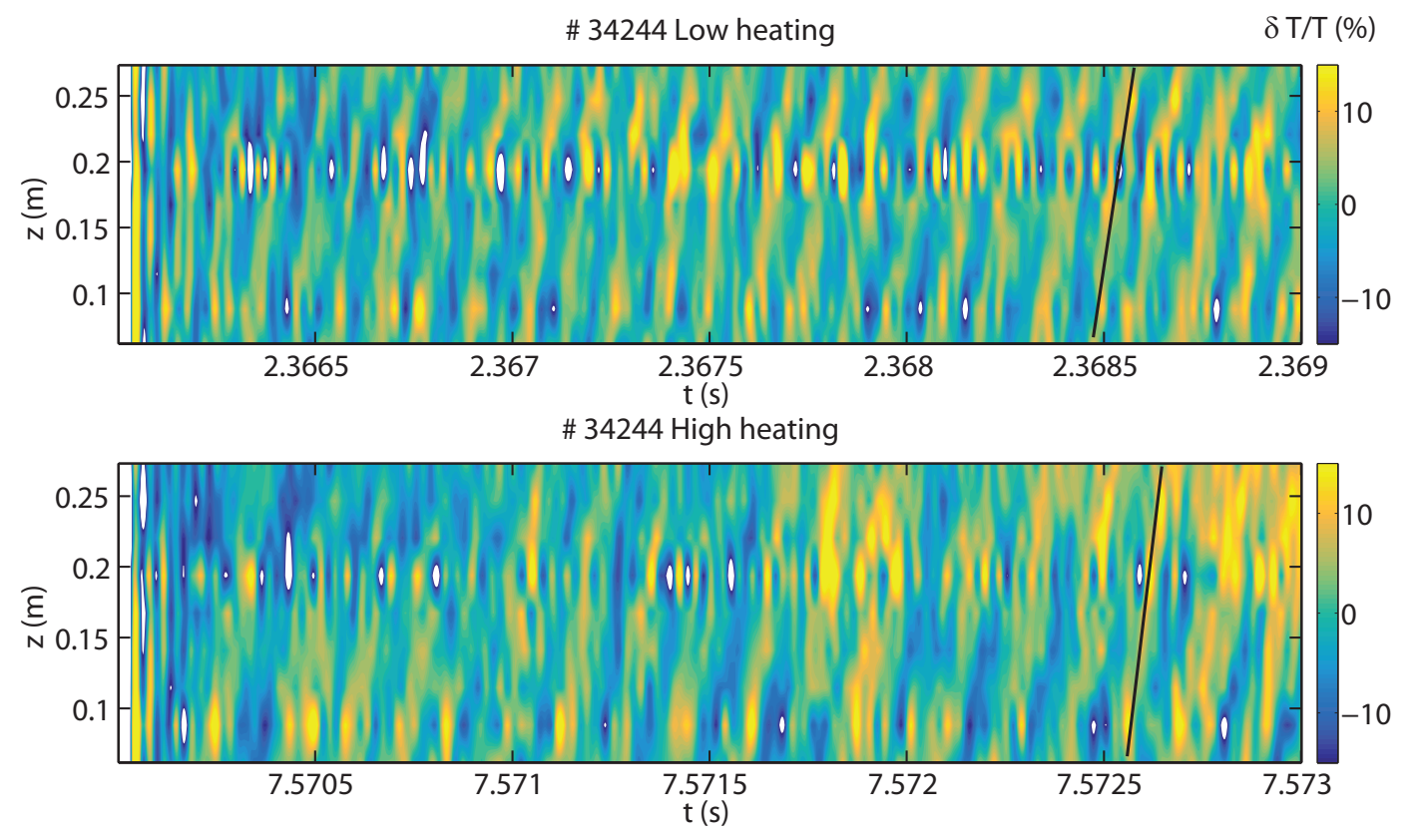

Figure 6. Time traces of the ECEI channels distributed poloidally along a single flux surface $\mathrm{q} \sim 5.5$ for discharge \#34244. The solid black lines mimic the inclination of the stripes representing the mode moving in the direction from the bottom to the top of the ECEI observation window.

0.97 and 0.99 , is over-plotted as the gray shaded vertical area. The horizontal shaded area corresponds to the mode velocity in the range of $1.5-3 \mathrm{~km} / \mathrm{s}$, measured with the ECE Imaging diagnostic. The cross-section of the two shaded areas corresponds to the region where the $E \times B$ velocity matches the measured mode velocity of $v_{E \times B}$ within the uncertainties. Thus, the combination of the two measurements: one from the ECEI and another of the $E_{\mathrm{r}}$ profile, allows for better determination of the position of the mode, which in our case would be in the upper half of the gradient region, closer to the pedestal top. In both cases the mode moves with the poloidal background flow $\left(v_{\text {mode }}=v_{\mathrm{E} \times \mathrm{B}}\right)$ and does not have a significant (measurable) intrinsic phase velocity $\left(v_{\mathrm{ph}} \sim 0\right)$. A poloidal wavelength for both cases is estimated as the distance between minimum and maximum amplitude of $\delta T / T$ shown in figure 9 . In the low heating phase, a poloidal wavelength is estimated to be $\lambda_{\text {pol }}=15 \pm 0.4 \mathrm{~cm}$, and in the case of the higher heating $\lambda_{\text {pol }}=24 \pm 0.4 \mathrm{~cm}$. This change in the poloidal wavelength is in-line with $50 \%$ change in frequency. The $q$ profiles for the two heating phases are shown in figure 8 . Thus, for the first phase where the toroidal mode number is $\mathrm{n}=13$ and $\mathrm{q}=5.6$, the poloidal mode number, calculated as $m=q \cdot n$, is $\mathrm{m}=73$. For the second phase, with $\mathrm{n}=14$ and $\mathrm{q}=5.55$, the poloidal mode number changes to $\mathrm{m}=78$. Taking into account the straight field line angle for the same equilibrium as for the phase I, from the divergence of the field lines in the poloidal direction at the outer midplane, the expected poloidal blob size is about $8 \mathrm{~cm}$ at the mode location. The poloidal wavelength is about $16 \mathrm{~cm}$. 


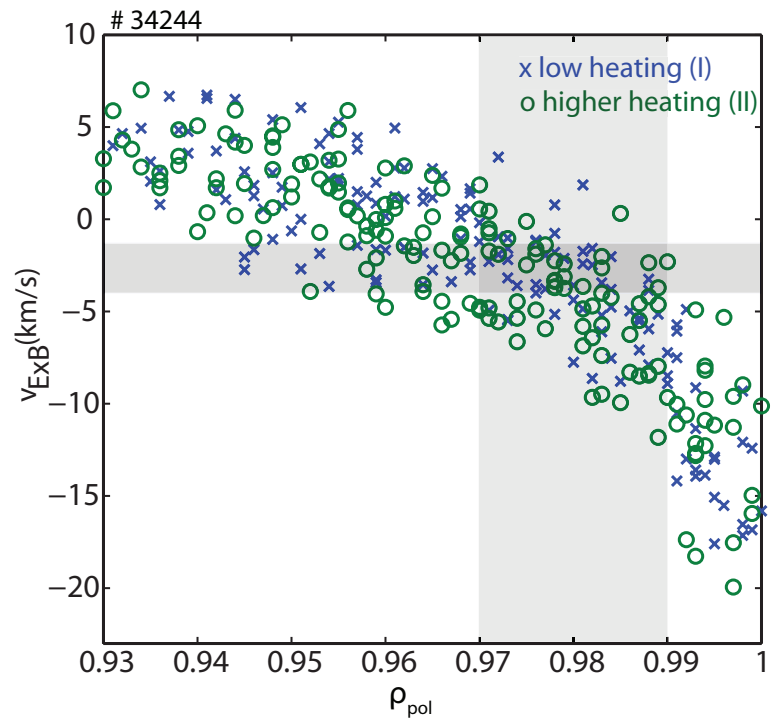

Figure 7. ELM synchronized $\mathrm{E} \times \mathrm{B}$ velocity measured with the edge Charge Exchange Recombination Spectroscopy diagnostic for two different heating phases, phase I and phase II, shown in figure 1.

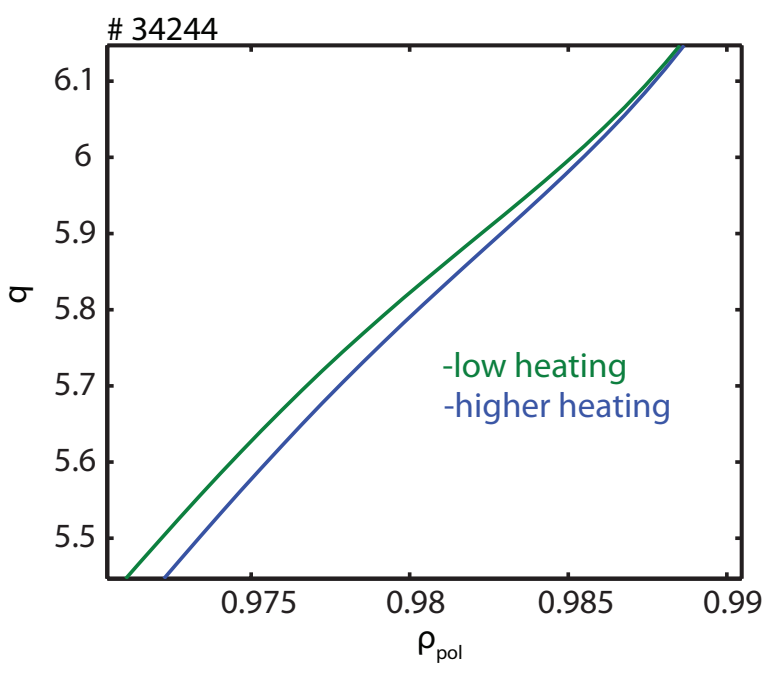

Figure 8. The $q$ profile for two different time points of the discharge \#34244. The profile corresponding to the phase of low heating shown in blue and the one during the higher heating phase, shown in green. Zoom into the region of the mode location.

This matches the experimental findings quite well.

A $50 \%$ change in mode frequency between the two heating phases is observed (see figure $3)$ and the poloidal wavelength changes $\left(f=v_{\text {mode,pol }} / \lambda_{\text {pol }}\right)$. Given the uncertainty of the $E \times B$ measurements, it is not possible to determine whether the small change in measured mode velocity matches with the change in the $E \times B$ velocity. However, the $20 \%$ higher heating power introduces a higher torque and corresponding higher 
toroidal rotation velocity which can also be seen in a higher core mode frequency (see figure 3). Moreover, the toroidal mode number changes from $n=13$ to $n=14$, and the

poloidal wavelength changes from $16 \mathrm{~cm}$ to $24 \mathrm{~cm}$. It could be that the mode changes its position, which might still be within the uncertainties of measurements of the $E \times B$, mode velocity and its position.

\section{Interaction between inter-ELM modes}

As already shown, the location of the low-frequency mode is in the upper part of the gradient region towards the pedestal top in contrast to the high-frequency modes whose position is shown to correspond to the minimum of the $E_{\mathrm{r}}$ [7]. The mid-frequency modes are located even further outside - very close to the separatrix [17]. The pedestal top and the steepest gradient are separated by about $5 \mathrm{~mm}$; the different modes, therefore, are spatially very close to each other. If multiple modes spatially overlap, the probability for non-linear interaction between them increases. As the modes are observed in the magnetic signal, the auto-bicoherence analysis could show if there is an energy transfer between the modes (of certain frequency) located in the pedestal region. Auto-bicoherence is defined as:

$$
B I C\left(f_{1}, f_{2}\right)=\frac{\left\langle\left|B\left(f_{1}, f_{2}\right)\right|\right\rangle^{2}}{\left\langle\left|X\left(f_{1}+f_{2}\right)^{2}\right|\right\rangle\left\langle\left|X\left(f_{1}\right)^{2} X\left(f_{2}\right)^{2}\right|\right\rangle},
$$

where $B\left(f_{1}, f_{2}\right)$ is defined as:

$$
B\left(f_{1}, f_{2}\right)=X\left(f_{1}\right) X\left(f_{2}\right) X\left(f_{1}+f_{2}\right)^{*}
$$

For this test, we use the signal measured with the coil \#B31-40, located at the outboard midplane. We shown the bicoherence result of one additional discharge \#34245. Discharge \#34245 is a repetition of discharge \#34244. Examples of the bicoherence results are shown in figure 9 and figure 10. Figure 9 shows the bicoherence calculated for two single inter-ELM phases during the discharge \# 34245. As can be seen in figure 9(a) significant bicoherence between the triplet $\sim 15 \mathrm{kHz}, 140 \mathrm{kHz}$, and $155 \mathrm{kHz}$ is observed. It can also be noted that the maximum bicoherence corresponds to a somewhat lower frequency, $\sim 12 \mathrm{kHz}$ which is the frequency of the harmonic of the core mode that is not strongly visible in the magnetic signal. As noted earlier in the manuscript this can largely influence any conclusion as the harmonic of the core mode and the low-frequency mode are in very close proximity. During the next inter-ELM phase, shown in figure 9 (b), a coupling of the $10 \mathrm{kHz}$ mode to the high frequency $\sim 205 \mathrm{kHz}$ mode is observed, thus forming a triplet of frequencies $\sim 10,195$, and $205 \mathrm{kHz}$. In both cases, we observe coupling of the high frequencies to the zero component of the Fourier spectrum. We show another example of coupling of the high-frequency mode to the low-frequency mode, during the discharge \#34244, in figure 10.

Increased bicoherence suggests a non-linear coupling between high and lowfrequency branches detected in the magnetic signal during inter-ELM periods. The three wave coupling is observed for a few individual inter-ELM phases, and not for 

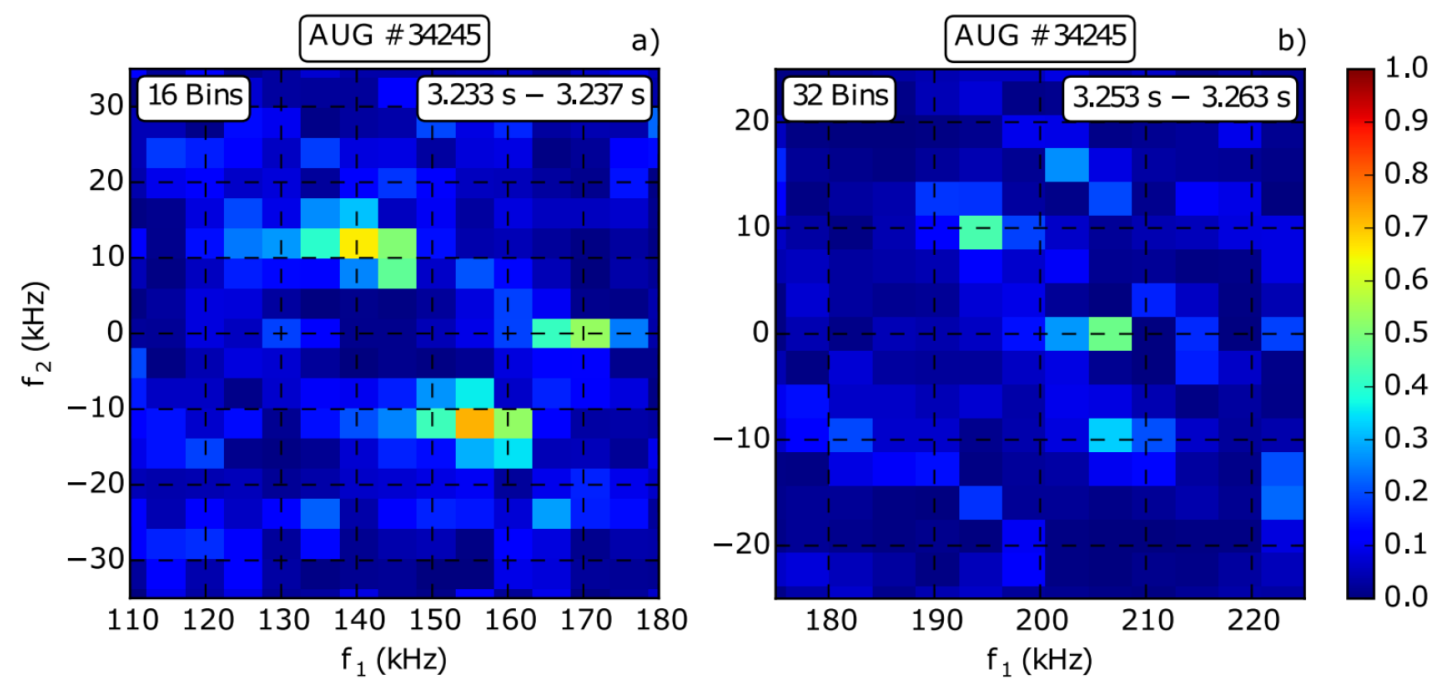

Figure 9. Bicoherence analysis for two different inter-ELM phases of single ELMs during the discharge \#34245. a) Coupling between mid and low frequency branches. b) Coupling between high and low frequency branches.

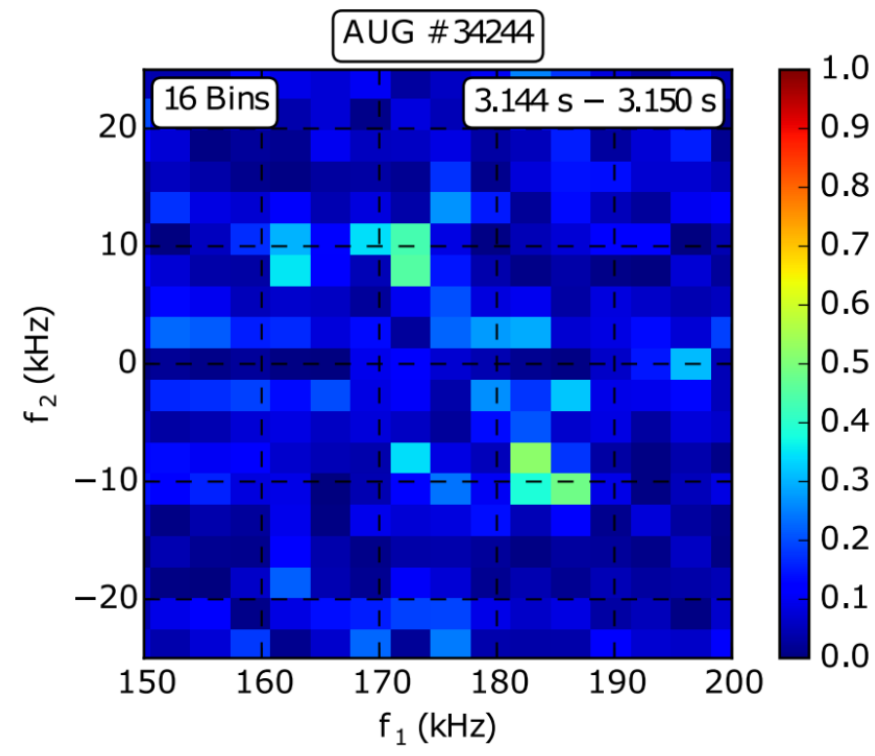

Figure 10. Bicoherence analysis for a single inter-ELM during the discharge \#34244.

all ELMs. One of the reasons could be that the low-frequency modes are not always visible in the $\dot{B}_{\mathrm{r}}$. The results showing a bicoherence for a few individual inter-ELM phases suggest a non-linear coupling between the observed modes in the region of the bad curvature region, however this does not always take place,and is therefore, not a necessary condition for the appearance of the modes. Quadratic mode coupling at the outer midplane has also been discussed in [34], although during an early stage of a non-linear ELM phase. 


\section{Summary and Discussion}

This work focuses on the characterization of low-frequency MHD modes appearing in between ELMs. They exist alongside other modes all of which can coexist in close spatial proximity. Their detection and full characterization are therefore not so straightforward. As noted, in the magnetic measurements they can also be hidden behind a strong core mode and its harmonics. The mode of interest appears at a low frequency with slowing down frequency patterns. We measure the low-frequency modes in discharges featuring low-frequency ELMs and long ELM cycles. The low frequency modes studied in this manuscript appear as the amplitude modulation of the radiation temperature $\left(T_{\text {rad }}\right)$, electron density $\left(n_{\mathrm{e}}\right)$, and the radial magnetic field $\left(\dot{B}_{\mathrm{r}}\right)$. ECE measurements show that the modes are localized in the upper half of the pedestal, unlike the high-frequency modes located in the region of steepest gradients [7]. For two cases shown in this manuscript, the frequency of the low frequency of modes inversely changes with input power, while the frequency of the high-frequency modes remains constant. This is also valid for all the other inter-ELMs within the two heating phases. The change in the frequency at higher heating power is attributed to the change in toroidal rotation and will be a subject of future work. Although the low and the high-frequency modes exist in different radial locations inside the plasma edge, a non-linear interaction is possible. This is seen in the bicoherence of the magnetic signal. Possibilities for coupling derive from the fact that the modes are spatially very close, and their spatial structure does not differ significantly ( $m$ numbers are found to be very similar). The velocity of lowfrequency modes matches the $E \times B$ velocity at the location of the modes within the uncertainties and does not significantly change for individual ELM cycles. The fact that the low-frequency modes appear alongside the high-frequency modes points towards the same or similar plasma conditions required for those to exist. As it has already been reported in [7], the high-frequency modes appear when the gradients are clamped to a fixed value. However, the low-frequency modes may also appear somewhat later in this phase. How the appearance of the low-frequency mode influences the pedestal evolution, and its stability remains an open question. Also, it remains unclear whether the modes are ideal or resistive. The studies made in Reference [35] explore the temperature perturbations associated with tearing modes. From this reference, we use the expression for the critical island width $\left(w_{\mathrm{c}}\right)$ that could cause a signature in the ECE data. The parallel heat conductivity is approximated by the Spitzer-Härm $\ddagger$ formula [36] and values for perpendicular heat conductivity are estimated from the experiment and varied within one order of magnitude (see Table 2). The critical island widths, $w_{\mathrm{c}}$, for a mode located at the pedestal top and toroidal mode number of $\mathrm{n}=13$, are shown in Table 2 . In order to cause a temperature modulation and increased local transport, the island width $w_{\mathrm{d}}$, has to be bigger then $w_{\mathrm{d}}>1.5 w_{\mathrm{c}}$ [37]. Only the larger island widths in this estimate would cause a phase jump in the ECE channels. The smaller ones cannot be resolved. From

$\ddagger \chi_{\|, S H}=3.6 \times 10^{29} \times \frac{T_{e}[\mathrm{keV}]^{5 / 2}}{n_{e}\left[\mathrm{~m}^{-3}\right]}\left[\mathrm{m}^{2} / \mathrm{s}\right]$ 


\begin{tabular}{|c|c|c|c|}
\hline$\chi_{\|}\left[\mathrm{m}^{2} / \mathrm{s}\right]$ & $2 \times 10^{7}$ & $1 \times 10^{8}$ & $5 \times 10^{8}$ \\
\hline$\chi_{\perp}\left[\mathrm{m}^{2} / \mathrm{s}\right]$ & & & \\
0.1 & $3.3 \mathrm{~mm}$ & $2 \mathrm{~mm}$ & $1.5 \mathrm{~mm}$ \\
0.3 & $4.3 \mathrm{~mm}$ & $2.9 \mathrm{~mm}$ & $1.9 \mathrm{~mm}$ \\
1 & $5.8 \mathrm{~mm}$ & $3.9 \mathrm{~mm}$ & $2.6 \mathrm{~mm}$ \\
\hline
\end{tabular}

Table 2. Calculated critical island widths $w_{\mathrm{c}}$, following [35]

the observations of the neighbouring ECE channels, we have seen that the amplitudes are in phase (see figure 5). This points toward an ideal mode rather than a tearing mode. However, further probing is planned using the correlation ECE diagnostics [38], with its better spatial resolution $(2-3 \mathrm{~mm})$ to clarify the possibility of an island at the pedestal top. The existence of such an island could prevent a pedestal top from growing further inwards, thus preventing an ELM from happening. A similar proposal on the existence of the 'wall' at the pedestal top has been made in [39] although in a different plasma scenario where ELM suppression is achieved with 3D fields. The two scenarios are not directly comparable, but the phenomenology of the mechanism could be similar. Nevertheless, the very accurate characterization of a low-frequency mode near the pedestal top, regarding its amplitude, location, poloidal and toroidal structure and velocity, can serve as a basis for comparison between experiment and non-linear modeling as done for example in [29].

\section{Acknowledgements}

G. F. Harrer is a fellow of the Friedrich Schiedel Foundation for Energy Technology. This work has been carried out within the framework of the EUROfusion Consortium and has received funding from the Euratom research and training programme 20142018 under grant agreement No 633053. The views and opinions expressed herein do not necessarily reflect those of the European Commission.

\section{References}

[1] F. Wagner, G. Becker, K. Behringer, D. Campbell, A. Eberhagen, W. Engelhardt, G. Fussmann, O. Gehre, J. Gernhardt, G. v. Gierke, G. Haas, M. Huang, F. Karger, M. Keilhacker, O. Kluber, M. Kornherr, K. Lackner, G. Lisitano, G. G. Lister, H. M. Mayer, D. Meisel, E. R. Muller, H. Murmann, H. Niedermeyer, W. Poschenrieder, H. Rapp, H. Rohr, F. Schneider, G. Siller, E. Speth, A. Stabler, K. H. Steuer, G. Venus, O. Vollmer, and Z. Yu. Regime of improved confinement and high beta in neutral-beam-heated divertor discharges of the ASDEX tokamak. Phys. Rev. Lett., 49:1408-1412, November 1982.

[2] H. Zohm. Edge localized modes (ELMs). Plasma Physics and Controlled Fusion, 38(2):105, 1996.

[3] J W Connor. Edge-localized modes - physics and theory. Plasma Physics and Controlled Fusion, 40(5):531, 1998.

[4] A. W. Leonard. Edge-localized-modes in tokamaks. Physics of Plasmas, 21(9):090501, 2014. 
[5] P. Gohil, M. Ali Mahdavi, L. Lao, K. H. Burrell, M. S. Chu, J. C. DeBoo, C. L. Hsieh, N. Ohyabu, R. T. Snider, R. D. Stambaugh, and R. E. Stockdale. Study of giant edge-localized modes in DIII-D and comparison with ballooning theory. Phys. Rev. Lett., 61:1603-1606, Oct 1988.

[6] A. Diallo, R. J. Groebner, T. L. Rhodes, D. J. Battaglia, D. R. Smith, T. H. Osborne, J. M. Canik, W. Guttenfelder, and P. B. Snyder. Correlations between quasi-coherent fluctuations and the pedestal evolution during the inter-edge localized modes phase on DIII-D. Physics of Plasmas, 22(5):056111, 2015.

[7] F M Laggner, E Wolfrum, M Cavedon, F Mink, E Viezzer, M G Dunne, P Manz, H Doerk, G Birkenmeier, R Fischer, S Fietz, M Maraschek, M Willensdorfer, F Aumayr, the EUROfusion MST1 Team, and the ASDEX Upgrade Team. High frequency magnetic fluctuations correlated with the inter-ELM pedestal evolution in ASDEX upgrade. Plasma Physics and Controlled Fusion, 58(6):065005, 2016.

[8] P. B. Snyder, H. R. Wilson, J. R. Ferron, L. L. Lao, A. W. Leonard, T. H. Osborne, A. D. Turnbull, D. Mossessian, M. Murakami, and X. Q. Xu. Edge localized modes and the pedestal: A model based on coupled peeling ballooning modes. Physics of Plasmas, 9(5):2037-2043, 2002.

[9] E. Wolfrum, E. Viezzer, A. Burckhart, M.G. Dunne, P.A. Schneider, M. Willensdorfer, E. Fable, R. Fischer, D. Hatch, F. Jenko, B. Kurzan, P. Manz, S.K. Rathgeber, and the ASDEX Upgrade Team. Overview of recent pedestal studies at asdex upgrade. Nuclear Fusion, 55(5):053017, 2015.

[10] M.N.A. Beurskens, L. Frassinetti, C. Challis, T. Osborne, P.B. Snyder, B. Alper, C. Angioni, C. Bourdelle, P. Buratti, F. Crisanti, E. Giovannozzi, C. Giroud, R. Groebner, J. Hobirk, I. Jenkins, E. Joffrin, M.J. Leyland, P. Lomas, P. Mantica, D. McDonald, I. Nunes, F. Rimini, S. Saarelma, I. Voitsekhovitch, P. de Vries, D. Zarzoso, and JET-EFDA Contributors. Comparison of hybrid and baseline elmy h-mode confinement in jet with the carbon wall. Nuclear Fusion, 53(1):013001, 2013.

[11] H.R. Koslowski, B. Alper, D.N. Borba, T. Eich, S.E. Sharapov, C.P. Perez, E. Westerhof, and JET-EFDA contributors. Observation of the palm tree mode, a new mhd mode excited by type-i elms on jet. Nuclear Fusion, 45(3):201, 2005.

[12] C P Perez, H R Koslowski, T C Hender, P Smeulders, A Loarte, P J Lomas, G Saibene, R Sartori, M Becoulet, T Eich, R J Hastie, G T A Huysmans, S Jachmich, A Rogister, F C Schller, and JET EFDA contributors. Washboard modes as elm-related events in jet. Plasma Physics and Controlled Fusion, 46(1):61, 2004.

[13] W Suttrop, K Bchl, H J de Blank, J Schweinzer, H Zohm, ASDEX Upgrade team, NBI group, and ICRH group. Characteristics of edge localized modes in ASDEX upgrade. Plasma Physics and Controlled Fusion, 38(8):1407, 1996.

[14] A L Colton, R J Buttery, S J Fielding, D A Gates, T C Hender, J Hugill, A W Morris, M Valovic, the COMPASS-D, and ECRH teams. Elm studies on the compass-d tokamak. Plasma Physics and Controlled Fusion, 38(8):1359, 1996.

[15] C.P. Perez, H.R. Koslowski, G.T.A. Huysmans, T.C. Hender, P. Smeulders, B. Alper, E. de la Luna, R.J. Hastie, L. Meneses, M.F.F. Nave, V. Parail, M. Zerbini, and JET-EFDA Contributors. Type-i elm precursor modes in jet. Nuclear Fusion, 44(5):609, 2004.

[16] T Bolzonella, H Zohm, M Maraschek, E Martines, S Saarelma, S Günter, and ASDEX Upgrade Team. High frequency MHD activity related to type I ELMs in ASDEX Upgrade. Plasma Physics and Controlled Fusion, 46(5A):A143, 2004.

[17] Felician Mink, Elisabeth Wolfrum, Marc Maraschek, Hartmut Zohm, Laszlo Horvath, Florian M Laggner, Peter Manz, Eleonora Viezzer, Ulrich Stroth, and the ASDEX Upgrade Team. Toroidal mode number determination of ELM associated phenomena on ASDEX upgrade. Plasma Physics and Controlled Fusion, 58(12):125013, 2016.

[18] J.E. Boom, I.G.J. Classen, P.C. de Vries, T. Eich, E. Wolfrum, W. Suttrop, R.P. Wenninger, A.J.H. Donne, B.J. Tobias, C.W. Domier, N.C. Luhmann Jr, H.K. Park, and the ASDEX Upgrade Team. 2D ECE measurements of type-I edge localized modes at ASDEX upgrade. 
Nuclear Fusion, 51(10):103039, 2011.

[19] P Manz, J E Boom, E Wolfrum, G Birkenmeier, I G J Classen, N C Luhmann Jr, U Stroth, and the ASDEX Upgrade Team. Velocimetry analysis of type-i edge localized mode precursors in asdex upgrade. Plasma Physics and Controlled Fusion, 56(3):035010, 2014.

[20] M. Kim, J. Lee, H.K. Park, G.S. Yun, W. Lee, C.W. Domier, Luhmann N.C. Jr., and KSTAR team. Multimode excitation during the inter-ELM-crash periods in KSTAR H-mode plasma. Nuclear Fusion, 55(7):073001, 2015.

[21] B Vanovac, S S Denk, E Wolfrum, M Willensdorfer, Wolfgang Suttrop, R Fischer, N C Luhmann, and the ASDEX Upgrade team. Mode analysis limitations of ECEI \& ECE measurements at the plasma edge. 20th Joint Workshop on ECE and ECRH, Greifswald, May 14-17, 2018 (to appear on EPJ Web of Conferences), 2018.

[22] B Vanovac, E Wolfrum, S S Denk, F Mink, F M Laggner, G Birkenmeier, M Willensdorfer, E Viezzer, M Hoelzl, S J Freethy, M G Dunne, A Lessig, N C Luhmann Jr, the ASDEX Upgrade Team, and the EUROfusion MST1 Team. Effects of density gradients and fluctuations at the plasma edge on ECEI measurements at ASDEX upgrade. Plasma Physics and Controlled Fusion, 60(4):045002, 2018.

[23] B. J. Tobias, M. E. Austin, J. E. Boom, K. H. Burrell, I. G. J. Classen, C. W. Domier, N. C. Luhmann Jr., R. Nazikian, and P. B. Snyder. ECE-imaging of the H-mode pedestal (invited). Review of Scientific Instruments, 83(10):10E329, 2012.

[24] Muhammad Khawar Ayub, Gunsu S. Yun, Woochang Lee, and Hyeon K. Park. Interpretation of the electron cyclotron emission signal from optically marginal plasmas. Journal of the Korean Physical Society, 70(3):268-275, 2017.

[25] A. Kallenbach, A. Carlson, G. Pautasso, A. Peeters, U. Seidel, and H.-P. Zehrfeld. Electric currents in the scrape-off layer in asdex upgrade. Journal of Nuclear Materials, 290-293(Supplement C):639 - 643, 2001. 14th Int. Conf. on Plasma-Surface Interactions in Controlled Fusion Devices.

[26] M Willensdorfer, G Birkenmeier, R Fischer, F M Laggner, E Wolfrum, G Veres, F Aumayr, D Carralero, L Guimarais, B Kurzan, and the ASDEX Upgrade Team. Characterization of the Li-BES at ASDEX upgrade. Plasma Physics and Controlled Fusion, 56(2):025008, 2014.

[27] W. Suttrop and A.G. Peeters. Practical limitations to plasma edge electron temperature mesurements by radiometry of electron cyclotron emission. IPP report, 1996.

[28] A Burckhart, E Wolfrum, R Fischer, K Lackner, H Zohm, and the ASDEX Upgrade Team. InterELM behaviour of the electron density and temperature pedestal in ASDEX Upgrade. Plasma Physics and Controlled Fusion, 52(10):105010, 2010.

[29] A.F. Mink, M. Hoelzl, E. Wolfrum, F. Orain, M. Dunne, A. Lessig, S. Pamela, P. Manz, M. Maraschek, G.T.A. Huijsmans, M. Becoulet, F.M. Laggner, M. Cavedon, K. Lackner, S. Gnter, U. Stroth, and The ASDEX Upgrade Team. Nonlinear coupling induced toroidal structure of edge localized modes. Nuclear Fusion, 58(2):026011, 2018.

[30] Denk, Severin S., Fischer, Rainer, Maj, Omar, Poli, Emanuele, Stober, Jorg K., Stroth, Ulrich, Vanovac, Branka, Suttrop, Wolfgang, Willensdorfer, Matthias, and ASDEX Upgrade Team. Radiation transport modelling for the interpretation of oblique ece measurements. EPJ Web Conf., 147:02002, 2017.

[31] S K Rathgeber, L Barrera, T Eich, R Fischer, B Nold, W Suttrop, M Willensdorfer, E Wolfrum, and the ASDEX Upgrade Team. Estimation of edge electron temperature profiles via forward modelling of the electron cyclotron radiation transport at ASDEX Upgrade. Plasma Physics and Controlled Fusion, 55(2):025004, 2013.

[32] R. Fischer, C. J. Fuchs, B. Kurzan, W. Suttrop, E. Wolfrum, and ASDEX Upgrade Team. Integrated data analysis of profile diagnostics at ASDEX upgrade. Fusion Science and Technology, 58(2):675-684, 2010.

[33] E. Viezzer, T. Putterich, G.D. Conway, R. Dux, T. Happel, J.C. Fuchs, R.M. McDermott, F. Ryter, B. Sieglin, W. Suttrop, M. Willensdorfer, E. Wolfrum, and the ASDEX Upgrade Team. Highaccuracy characterization of the edge radial electric field at asdex upgrade. Nuclear Fusion, 
53(5):053005, 2013.

[34] I. Krebs, M. Hlzl, K. Lackner, and S. Gnter. Nonlinear excitation of low-n harmonics in reduced magnetohydrodynamic simulations of edge-localized modes. Physics of Plasmas, 20(8):082506, 2013.

[35] Richard Fitzpatrick. Helical temperature perturbations associated with tearing modes in tokamak plasmas. Physics of Plasmas, 2(3):825-838, 1995.

[36] Lyman Spitzer and Richard Härm. Transport phenomena in a completely ionized gas. Phys. Rev., 89:977-981, March 1953.

[37] Matthias Hoelzl. Diffusive Heat Transport across Magnetic Islands and Stochastic Layers in Tokamaks. Dissertation, Technische Universität München, München, 2010.

[38] S. J. Freethy, G. D. Conway, I. Classen, A. J. Creely, T. Happel, A. Khn, B. Vanovac, and A. E. White. Measurement of turbulent electron temperature fluctuations on the ASDEX Upgrade tokamak using correlated electron cyclotron emission. Review of Scientific Instruments, 87(11):11E102, 2016.

[39] P. B. Snyder, T. H. Osborne, K. H. Burrell, R. J. Groebner, A. W. Leonard, R. Nazikian, D. M. Orlov, O. Schmitz, M. R. Wade, and H. R. Wilson. The EPED pedestal model and edge localized mode-suppressed regimes: Studies of quiescent H-mode and development of a model for edge localized mode suppression via resonant magnetic perturbations. Physics of Plasmas, 19(5):056115, 2012. 